\title{
Éditorial: Des situations de vieillissement
}

La rédaction d'un éditorial sur une série d'articles constitue tout un défi notamment celui de trouver le fil conducteur du travail d'un groupe d'auteurs qui ont écrit leurs articles indépendamment les uns des autres. Heureusement, la recherche d'un thème suscitant un débat sur les six textes du présent numéro n'était pas difficile. Tous portent sur les contextes du vieillissement.

Ce n'est que récemment que nous en sommes venus à juger important le cadre dans lequel vivent les aîné(e)s. ${ }^{1}$ Nos traditions se révèlent beaucoup plus naïves. Pendant longtemps, nous avons considéré le vieillissement comme une période de déclin inévitable. Des phrases du style «à quoi peux-tu t'attendre à ton âge» ou "on n'apprend pas à un vieux singe à faire la grimaces témoignaient de cette perception du vieillissement. Subséquemment, nos préoccupations au sujet du vieillissement se sont accompagnées de beaucoup d'optimisme concernant les possibilités qui viennent avec l'âge. L'émission "It's About Time» sur la chaine CBC a étayé cette question. Au cours de cette émission, on nous a présenté des portraits d'aînés qui s'occupent à faire de la nage aérienne, à escalader des montagnes, à courir des marathons et à gérer des entreprises multinationales. Des phrases du style "plus on vieillit, meilleur on est» caractérisent cette compréhension du vieillissement. Cependant, il est clair qu'aucune de ces images ne reflétaient la vie de plusieurs aînés canadiens.

Nous admettons maintenant que les aînés constituent un groupe hétérogène de personnes qui ont derrière elles six décennies ou plus d'expériences diverses et de temps sur lesquels fonder leurs valeurs, leurs croyances et leur idiosyncrasie. Ils diffèrent beaucoup plus entre eux que les personnes de tout autre groupe d'âge. Et nous commençons seulement à explorer la complexité de ces différences. L'examen de l'interaction entre les aînés et les différents environnements dans lesquels ils vivent constitue une approche d'examen de cette diversité.

L'approche écologique pour comprendre l'expérience et le comportement humains constitue le paradigme qui alimente ma recherche et mon enseignement en écologie humaine. ${ }^{2}$ Certaines des hypothèses de ce paradigme reposent sur le fait que les personnes vivent dans un environnement physique et interpersonnel donné; ces personnes sont influencées et, en retour, elles ont une influence sur cet environnement; limportance relative de l'environnement varie selon les individus et se modifie dans le temps pour un même individu. 
Pendant plusieurs années, j'ai songé à la façon de conceptualiser les environnements principaux des ainés. Je vois six environnements ou contextes qui sont particulièrement importants pour comprendre la vie des aînés. On peut voir ces environnements comme des cercles concentriques dont les plus rapprochés ont une incidence plus directe sur les aînés. Au centre, se trouve l'environnement personnel, notamment les caractéristiques de la personne, à savoir ses capacités fonctionnelles et cognitives et ses ressources personnelles comme le revenu. Ces caractéristiques fournissent des possibilités et des contraintes d'interaction avec d'autres environnements. Vient ensuite l'environnement «immédiat» qui comprend les attributs du lieu où la personne demeure et les objets du lieu. L'environnement interpersonnel comprend les personnes importantes avec lesquelles la personne interagit, y compris les membres de son réseau social et de soins de santé. L'environnement physique inclut tant l'environnement architectural que l'environnement naturel à l'extérieur du domicile. Le climat canadien rigoureux constitue une caractéristique de cet environnement. L'environnement culturel inclut les croyances au sujet du vieillissement et la place des aînés dans la population. Finalement, l'environnement politique regroupe l'ensemble des politiques et des programmes qui peuvent influencer les relations des aînés avec tout autre environnement. Les articles du présent numéro dénotent un intérêt pour ces différents contextes.

Dans son article sur l'interaction entre les aînés qui vivent dans des logements privés pour aînés et leurs environnements physique et interpersonnel, Maltais parle de la question du «meilleur ajustement» entre les ressources personnelles des aînés et leurs environnements. Elle s'intéresse à la façon dont les environnements architectural et interpersonnel (au sens de soins) peuvent influer sur l'intégration sociale des résidants. Maltais relève plusieurs ressources environnementales qui peuvent influer sur l'intégration sociale. On peut voir le niveau des services fournis comme un certain support aux environnements physique et de service. Les perceptions qu'ont les résidants de leur contrôle environnemental reflètent la croyance qu'ils (ou elles) peuvent avoir une influence sur leur environnement immédiat, alors que le niveau de tolérance des soignants à l'égard des aînés et leur participation aux décisions reflètent l'aide qui provient des environnements interpersonnel ou de soins.

Maltais est d'avis que le niveau de services et la perception du contrôle environnemental constituent les indicateurs prévisionnels les plus importants de l'intégration sociale et qu'il y a une interaction entre les environnements physique et personnel. Pour les aînés dont le niveau d'habiletés fonctionnelles est élevé, avoir un contrôle sur leur environnement est encore plus important alors que pour ceux qui sont fragiles, trop de contrôle sur leur environnement constitue un stress. Elle conclut que les aînés ont besoin de différents environnements organisationnels selon leur niveau de 
fragilité, un argument en faveur d'un «ajustement meilleur» entre les ressources personnelles et les autres ressources environnementales.

Le principal objet de l'article de Wielink et Huijsman est l'environnement de soins. Ils ont étudié les attitudes des personnes de plus de 65 ans qui résident dans la communauté et reçoivent des soins non structurés par rapport à ceux qui reçoivent des soins structurés dans des situations de "besoins impérieux": aide à l'entretien ménager et soins personnels à court et à long terme. Les auteurs ont découvert que lorsque les besoins de soins augmentent, les aînés ont tendance à préférer l'aide structurée même si leurs préférences sont influencées par une combinaison de facteurs: leur expérience de prestations de soins, leur sexe et la composition de la maisonnée. Dans l'ensemble, les soins structurés et non structurés sont vus comme des détracteurs face à leur indépendance étant donné que tous deux sont perçus comme les manifestations d'une dépendance croissante. Ainsi, se retrouver en situation de recevoir des soins est un indice d'habiletés réduites qui influence l'environnement d'une personne.

Dans leurs conclusions, Wielink et Huijsman font valoir que les préférences des consommateurs aînés auront une grande influence sur le type de services utilisés. Ils examinent la question de savoir si la correspondance entre les désirs des aînés et la disponibilité des ressources devrait se modeler par la modification des préférences des aînés ou par celle des ressources de soins pour répondre à ces préférences. De plus, ils laissent entendre que ces questions doivent avoir une incidence sur le développement des politiques liées aux soins de longue durée pour les aînés.

Robb, Denton, Gafni, Joshi, Lian, Rosenthal et Willison examinent l'environnement interpersonnel des aînés en étudiant leurs contributions à l'aide aux personnes qui font partie de leurs réseaux informels. Leur article porte sur la valeur économique de cette contribution qui devrait autrement être monnayée sur le marché. Les auteurs ont découvert que les aînés contribuent plus que les jeunes à l'aide non monnayée. Selon toute vraisemblance, leur participation est presque le double en ce qui a trait à l'aide structurée et leur engagement dans l'aide non structurée compte pour une fois et demie de plus. De fait, ils fournissent presque la moitié de l'ensemble des activités de soutien non rémunérées au Canada. En comparant les aînés aux autres aidants naturels, Robb et al. nous rappellent que si nous considérons les aînés uniquement comme des personnes qui reçoivent des ressources, nous ne tenons jamais compte de leurs contributions.

L'article d'Hallman et de Joseph met l'accent sur les environnements physique et géographique liés à la prestation des soins. Plus particulièrement, ils se prononcent sur la question de la proximité par rapport à la somme et la nature de la participation aux soins non structurés aux aînés. Ils font ressortir une réalité concernant la prestation des soins au Canada: dans la plupart des cas, on doit franchir une barrière. Hallman et Joseph 
fournissent la preuve que les questions d'espace-temps diffèrent pour les femmes et les hommes lorsqu'ils fournissent des soins et ils concluent que les femmes semblent accorder moins d'importance aux distances que les hommes en matière de soins. Ils ont découvert que les femmes soignantes voyagent plus et plus longtemps que les hommes pour fournir des soins et sont plus proactives que les hommes lorsqu'elles conjuguent avec des problèmes de distance, en organisant leur déménagement et celui de leurs proches. Cependant, ils ont aussi découvert que bien que les femmes semblent plus proactives dans leur façon de conjuguer avec les contraintes d'espace-temps, c'est en travaillant plus fort et plus vite qu'elles y arrivent. Les auteurs sont d'avis que la prochaine étape dans la compréhension des soins de santé aux aînés en regard du sexe et de la géographie consiste à passer d'une analyse dyadique à une analyse des réseaux familiaux de soignants auprès des proches qui sont plus âgés, pour ainsi examiner la "géographie de la famille».

Dans ce numéro, deux articles examinent des questions culturelles, bien que de façon fort différente. Gee écrit au sujet d'un aspect de l'environnement culturel des aînés, leur identité ethnique, alors que la recherche de Connidis et de McMullin se centre en partie sur l'aspect culturel de l'édification des croyances en ce qui a trait aux familles.

Dans l'introduction de sa recherche sur l'identité ethnique des aînés canadiens nés en Chine, Gee remarque que peu de recherches ont porté sur l'étude du vieillissement chez les ethnies au Canada. Elle prétend que les aînés chinois canadiens n'expérimentent pas un vieillissement normal dans le contexte canadien. Ils sont plutôt «en train de forger leur expérience de vieillissement en contexte canadien qui, pour la première fois, leur offre un environnement social moins contraignant et moins édifié sur des politiques d'immigration racistes" (p. 5). Pour la plupart d'entre eux, cette identité est canadienne. Seule une minorité de participants à son étude ont conservé leur identité chinoise. Pour ceux-ci, le rejet de l'identité de la majorité culturelle semble avoir un effet négatif. Loin d'être entourés de relations avec ceux qui ont des expériences similaires, ils perçoivent comme inférieurs leur niveau de santé et le soutien social dont ils bénéficient.

Gee conclut que nos connaissances au sujet du contexte sont encore limitées et que «les sociologues ne peuvent simplement vanter les mérites de la conservation de l'identité ethnique malgré l'actuelle adulation théorique de la différence» (p. 18).

Connidis et McMullin abordent la question de savoir si ceux qui n'ont pas d'enfants acceptent le point de vue sociétal selon lequel les gens sans enfants risquent fort d'être marqués par la solitude et l'isolement. Ils s'appuient sur une étude d'entrevues auprès d'ainés sans enfants. La majorité de ces aînés ont déclaré tant les avantages, soit avoir moins de soucis et être plus à l'aise financièrement que les désavantages, soit le manque de compagnonnage et la solitude. Il est intéressant de constater que ceux qui déclarent se préoccuper de la solitude et de l'isolement ont, 
dans leur vie, des niveaux de satisfactions similaires à ceux qui ne mentionnent pas ces désavantages. Parallèlement, ceux qui se préoccupent du manque de soutien et d'accessibilité aux soins non structurés lorsqu'ils en ont besoin ont des liens sociaux de même type que ceux qui déclarent ne pas avoir ce genre de préoccupations. Les auteurs concluent que ceux qui n'ont pas d'enfants ont accepté certains des stéréotypes liés au fait de ne pas avoir d'enfants.

Les auteurs des articles de ce numéro fournissent un aperçu de l'état de notre compréhension de la façon dont les aînés influencent les environnements dans lesquels ils vivent et sont influencés par eux. Le concept de congruence entre les valeurs, les préférences et les ressources personnelles des aînés et leurs environnements principaux constituent la trame de leurs travaux. À mon point de vue, nous avons besoin d'aller de l'avant avec le concept d' "ajustement meilleur» pour nous repositionner. Si nous acceptons le paradigme que les ainés sont des agents dans leur vie, alors nous devons centrer nos recherches sur la façon dont leurs environnements favorisent ou détruisent leurs habiletés à s'engager dans la vie aussi pleinement que possible. De plus nous ne devons pas oublier que l' «ajustement) entre les besoins des aînés et leurs environnements n'est pas statique et qu'on doit l'examiner dans le cadre de l'évolution des environnements. La Revue canadienne du vieillissement fournit une excellente tribune à ce genre de propos qui s'enrichissent d'informations provenant d'approches méthodologiques et d'antécédents disciplinaires multiples, comme ceux que l'on retrouve dans le présent numéro.

\section{Notes}

1 Le masculin embrasse le féminin.

2 Voir, par example, Keating, Fast, Connidis, Penning, et Keefe (1997).

\section{Reference}

Keating, N., Fast, J., Connidis, I., Penning, M., \& Keefe, J. (1997). Bridging policy and research in elder care. La Revue canadienne du vieillissement/Analyse de politiques Édition spéciale mixte, 16(Supp.), 22-41. 